\title{
Experimental Progress in the Direct Detection of Dark Matter
}

\author{
Nigel J.T. Smith \\ Particle Physics Department, CCLRC Rutherford Appleton Laboratory, \\ Chilton, Didcot, Oxon, OX11 OQX, U.K.
}

\begin{abstract}
Several experimental approaches are being utilised for the direct detection of the hypothetical weakly interacting massive particle which may constitute the majority of the non-luminous component of the Galaxy. These experimental techniques exploit the coherent recoil of target nuclei during an interaction and include thermal, ionisation, scintillation and tracking detectors. The challenges associated with this detection, the techniques employed and the current status of these searches are reviewed.
\end{abstract}

\section{Introduction}

A standard cosmological model of the Universe has been evolving over the last few years from observational data collected at multiple wavelengths, incorporating different epochs of the Universe and different physics principles. One of the consequences of this emergent 'convergence' model is that up to $90 \%$ of the matter in the Universe is in the form of non-relativistic, non-baryonic, non-luminous material (Spergel, 2003). One possible solution to this cold dark matter problem, supported by theoretically favoured supersymmetric extensions to the standard model of particle physics, is that this material comprises Weakly Interacting Massive Particles (WIMPs). Examples of such particles are the neutralino of the CMSSM (Ellis et al, 2003), the lightest stable admixture of the neutral supersymmetric particles, with expected masses in the $100 \mathrm{GeV}$ range.

The direct observation of a neutralino population within the Galaxy would impart information to the fields of cosmology and astrophysics allowing refinement and confirmation of the current standard paradigm. Within particle physics, such an observation would also provide complementary information to accelerator of supersymmetric models through investigation of spin dependence, target mass dependence and, uniquely, testing R-parity conservation a multiplicative quantum number prohibiting the decay of supersymmetric particles.

The Galactic component of this cold dark matter may be directly observed through the coherent elastic scattering off target nuclei. This is a near-maximal energy transfer process due to the comparable masses of the dark matter WIMP and the target nuclei in many detector systems (Smith \& Lewin, 1990).

\section{Dark Matter detector requirements}

The expected scalar interaction cross section for neutralino dark matter elastic scattering is in the range $10^{-8} \mathrm{pb}$ to $10^{-10} \mathrm{pb}$ (Ellis et al, 2003). This trans- 
lates to an expected event rate in a target material of between 0.01 and 0.0001 events $/ \mathrm{kg} /$ day, i.e. of order events/tonne/week for the lower bound. The expected recoil energy deposition for a WIMP interaction is below $50 \mathrm{keV}$, with an exponential energy spectrum. The low interaction rate and small energy deposition require detectors with high signal to noise capability (better than $10^{5}$ ), low energy threshold (keV), ultimately high mass (tonne scale), and low intrinsic background levels (event $/ \mathrm{kg} /$ day of target). A low energy threshold also reduces the effect of the form factor introduced in the coherent scattering process.

The key requirement for direct dark matter detectors, following maximal background reduction, is the ability to differentiate the nuclear recoils initiated by WIMP collisions and the more frequent electron recoils due to gamma and beta backgrounds. In addition nuclear recoils due to neutrons must be clearly quantified and sufficient shielding or active veto be in place to reduce these events well below the expected WIMP rate. These neutrons arise from $(\alpha, n)$ reactions from uranium and thorium impurities in the experimental cavern walls, detector shielding and detector components, as well as muon spallation neutrons from these regions generated by the high energy cosmic ray muons that penetrate to the detector cavern. Position sensitivity is a characteristic of the new generation of dark matter detectors, which will allow the rejection of internal background through the definition of a fiducial volume, and the rejection of neutrons through segmentation and multiple scattering.

Ultimately the use of targets with different mass and spin components, or different targets and techniques, will be required to provide information about the characteristics of the WIMP nucleon interaction. The use of multiple targets also provides cross checks on systematics and backgrounds due to the $A^{2}$ dependence of the scalar interaction rate of the WIMPs as opposed to the $A$ dependence on neutron scattering.

An alternative approach to the nuclear recoil discrimination is the use of directional information to correlate the motion of the Earth through the Galactic dark matter halo, or the modulation of the signal due to the annual or diurnal variations due to the Earth's rotation. This technique provides the ability to perform dark matter halo studies within the local environment and will be complementary to indirect searches where integration of the WIMP flux occurs in the Galactic centre or Sun.

\section{Dark Matter detectors}

Many experiments are currently being undertaken to search for WIMP interactions, using many different detector technologies (Morales, 2003). All detector systems achieve low background levels through the use of radio-pure materials in construction, radio-pure gamma shielding of the detector from the surroundings and operation of the detector at depth to reduce the effect of cosmic rays. Detector technologies currently affording the best limits on the scalar WIMP cross sections, at about $10^{-6} \mathrm{pb}$, are based on two technologies cryogenic semiconductor devices and scintillation based detectors.

The EDELWEISS (Benoit et al, 2002) and CDMS (Schnee et al, 1998) collaborations use detectors based on cryogenic semiconductor devices where the ionisation and phonons created in the target during a particle interaction 

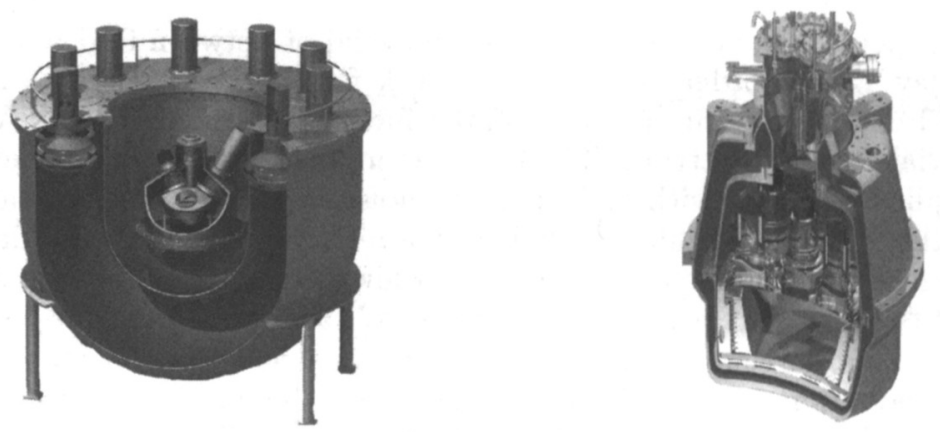

Figure 1. Cut-away diagrams showing examples of direct dark matter detection targets using liquid xenon in scintillation and scintillation/ionisation mode.. The ZEPLIN I and ZEPLIN II liquid xenon targets are operated at the Boulby mine to reduce muon initiated backgrounds. Left, ZEPLIN I - a $5 \mathrm{~kg}$ chamber of liquid xenon is viewed by three photomultipliers through $3 \mathrm{~cm}$ liquid xenon turrets. The target is surrounded by a 1 tonne liquid scintillator veto to reject Compton scattered high energy gamma from the PMTs, and a lead castle, not shown. Right, ZEPLIN II - a two phase chamber where the ionisation from the interaction is extracted into the gas phase and observed through electroluminescence in a high field region.

are observed. The ratio of these two signal channels provides a powerful method of discrimination between the WIMP induced nuclear recoils and background induced electron recoils.

The ZEPLIN collaboration (Smith et al, 2003) is developing a series of detector based on liquid xenon (shown in Fig. 1). ZEPLIN I (Barton et al, 2003) is a single phase detector and utilises the scintillation light produced in particle interactions. The time constant of emission of this light is used as a statistical discriminant between nuclear and electron recoils. Future generations of these detectors will utilise two phase operation where the ionisation created in the interaction is also measured, through extraction into the gas phase and observation through electroluminescence, providing improved discrimination.

The DAMA collaboration (Bernabei et al, 2000) utilise a $100 \mathrm{~kg}$ array of nine $\mathrm{NaI}$ detectors, again measuring the scintillation light produced during an interaction. Although photomultiplier noise pulses are rejected based on the time constant of the light observed, the WIMP identification strategy utilised in this experiment is to search for the expected annual modulation as the Earth rotates around the sun within the WIMP halo. Such an annual modulation has been observed over four years and has been interpreted as a WIMP signature in the absence of other variable backgrounds (Bernabei et al, 2000).

\section{Current status}

The current status of direct dark matter searches is shown in Fig. 2 produced from the web based limit plotter provided by Gaitskel \& Mandic (2003). For spin dependent interactions only $90 \%$ c.l. upper limits are shown for various experiments, with references summarised by Gaitskel \& Mandic. For spin independent 

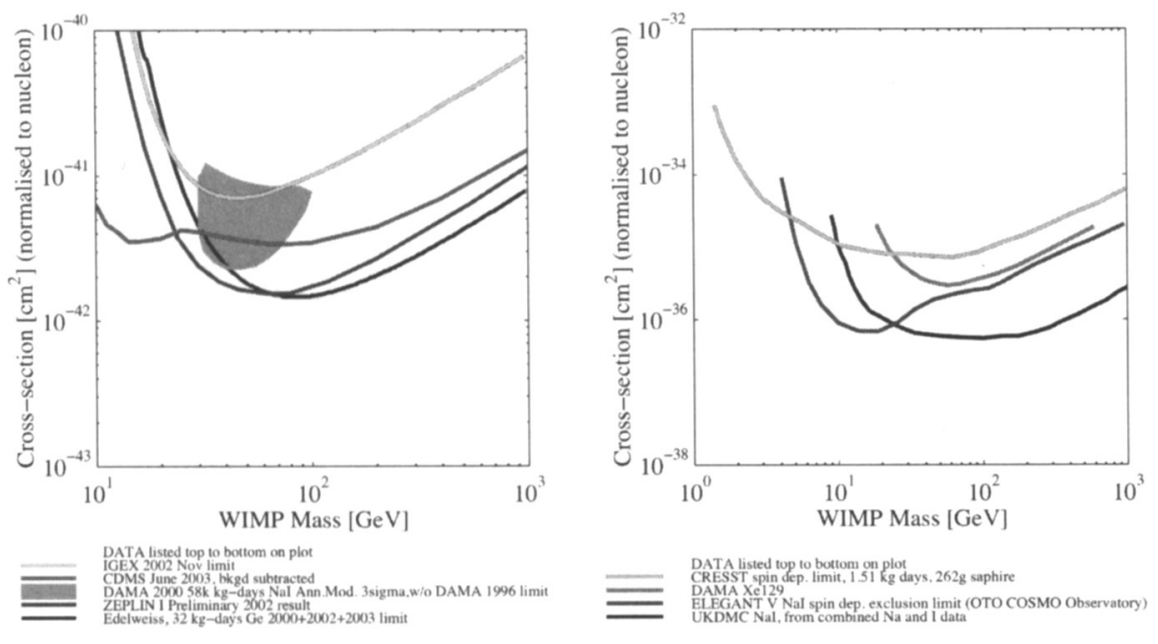

Figure 2. Current status of dark matter searches, shown as WIMP-nucleon cross section vs. WIMP mass. Left diagram shows spin independent, i.e. scalar, interactions, whilst right diagram shows spin dependent cross section, both normalised to one nucleon.

interactions the solid region is the interpretation of the DAMA annual modulation as a WIMP signature based on a fully spin independent interaction. The curves are $90 \%$ c.l. upper limits set by the experiments discussed above, where no signal is detected. For comparison, current supersymmetric models predict a range of interactions between $10^{-8} \mathrm{pb}$ to $10^{-10} \mathrm{pb}$ in the spin independent case.

\section{References}

Barton J. C. et al, 2003, Workshop on Identification of Dark Matter (York) 302

Benoit, A. et al, 2002, Phys. Lett B, 545, 43

Bernabei R. et al, 2000, Phys. Lett. B, 480, 23

Ellis, J. R. et al, 2003, Phys. Lett B, 565, 176

Gaitskel R., Mandic V., 2003 http://dendera.Berkeley.EDU/plotter/entryform.html

Morales, A., 2003, Nucl. Phys. Proc. Suppl. 114, 39

Schnee, R. et al, 1998, Phys. Rep. 307, 283

Smith, P. F., Lewin J.D. 1990, Phys. Rep. 187, 203

Smith, N. J. T. et al, 2002, Heidelberg Conference (Cape Town) 499

Spergel D. N. et al, 2003, ApJS, 148, 175 